\title{
Carotid Intima-Media Thickness is a Predictor of Subclinical Myocardial Damage in Men with Type 2 Diabetes Mellitus
}

\author{
Authors \\ Sebastian Hörber 1, 2, 3, Angela Lehn-Stefan², 3, Anja Hieronimus'2, 3, 4, Sarah Hudak 2, 3, 4, Louise Fritsche2, 3, 4, \\ Andreas Fritsche 2, 3, 4D, Martin Heni², 3, 4, Hans-Ulrich Häring 2, 3, 4, Andreas Peter 1, 2, 3, Elko Randrianarisoa 2, 3
}

\section{Affiliations}

1 Department for Diagnostic Laboratory Medicine, Institute for Clinical Chemistry and Pathobiochemistry, University Hospital Tübingen, Germany

2 Institute for Diabetes Research and Metabolic Diseases of the Helmholtz Center Munich at the University of Tübingen, Germany

3 German Center for Diabetes Research (DZD), MünchenNeuherberg, Germany

4 Department of Internal Medicine IV, Division of Endocrinology and Diabetology, Nephrology University Hospital of Tübingen, Germany

Key words

High-sensitivity cardiac troponin I, type 2 diabetes mellitus, intima-media thickness, cardiovascular disease

$\begin{array}{ll}\text { received } & 23.10 .2019 \\ \text { revised } & 08.01 .2020 \\ \text { accepted } & 27.01 .2020\end{array}$

published online $\quad 04.03 .2020$

Bibliography

Exp Clin Endocrinol Diabetes 2021; 129: 750-756

DOI 10.1055/a-1107-2657

ISSN $0947-7349$

(c) 2020. Thieme. All rights reserved.

Georg Thieme Verlag KG, Rüdigerstraße 14, 70469 Stuttgart, Germany

Correspondence:

Andreas Peter, MD

Hoppe-Seyler-Straße 3

72076 Tübingen

Germany

Tel. + 49-7071-2985664, Fax: + 49-7071-294690

andreas.peter@med.uni-tuebingen.de

\section{ABSTRACT}

Background Type 2 diabetes mellitus (T2DM) promotes the development of atherosclerosis and is a major risk factor for cardiovascular disease. High-sensitivity cardiac troponin I (hscTnl) assays fundamentally improved the diagnosis of myocardial injury and even enable the prediction of future cardiovascular events in the general population. However, data about the association of hs-cTnl with cardiovascular risk factors and carotid intima media thickness (cIMT) as a marker of atherosclerosis are limited, especially in patients with T2DM.

Methods In this cross-sectional study we analyzed clinical and laboratory parameters of 234 patients ( $43 \%$ women) with T2DM and a median age of 65 years (interquartile range: 57 71). The median duration of diabetes mellitus was 10 years (6-17). Anthropometric data, blood pressure, glycemic parameters and lipid profiles were determined. Hs-cTnl plasma concentrations were measured on an ADVIA Centaur XPT immunoassay analyzer and cIMT was evaluated by high-resolution ultrasound.

Results Hs-cTnl plasma concentrations were below the gender-specific $99^{\text {th }}$ percentile in $93 \%$ of T2DM patients with a median concentration of $4.0 \mathrm{ng} / \mathrm{I}$ (interquartile range: 2.0 10.0). Hs-cTnl was significantly associated with gender, renal function and C-reactive protein in the entire study cohort. Gender-specific analyses revealed cIMT and renal function to be significantly associated with hs-cTnl in men. Contrary, only age was significantly associated with hs-cTnl in women.

Conclusion In a real-world clinical setting in patients with T2DM, cIMT is a predictor of subclinical myocardial damage in men, but not in women.

\section{Introduction}

Type 2 diabetes mellitus (T2DM) promotes the development of atherosclerosis thereby increasing the risk for cardiovascular disease (CVD), the leading cause of morbidity and mortality in T2DM [1, 2]. Compared with persons without diabetes, patients with T2DM have a twofold higher risk developing CVD and diabetes-related macrovascular complications. This atherogenic risk is known to be markedly larger in men compared to women $[3,4]$. Nowadays, the combination of clinical markers with biomarkers allows the identification of individuals at increased CVD. This can enable appropriate 
treatment tailored to patients with an already existing macrovascular disease, such as coronary heart disease. Cardiac-specific troponins (cTn) are powerful biomarkers to detect subclinical myocardial damage and revolutionized treatment in patients with acute coronary syndrome (ACS) [5-7]. Subclinical myocardial damage leads to release of cardiac troponin I (cTnI) and T (cTnT) that are detectable from the bloodstream. The development and introduction of high-sensitivity (hs) assays for the determination of cTn not only fundamentally improved the diagnostic performance and clinical utility, but also allowed the quantification of very low cTn concentrations seen in asymptomatic individuals and the detection of subclinical myocardial damage in individuals without overt acute cardiac disease $[8,9]$. The term high-sensitivity is defined as the ability to measure troponin concentrations in $>50 \%$ of a healthy reference population with a coefficient of variation $<10 \%$ at the $99^{\text {th }}$ percentile [10-12]. Besides the tremendous role in the management of patients with ACS, recent studies demonstrated prognostic implications of hs-cTn determination in individuals without acute cardiac morbidities [13-16]: Hs-cTn is recognized as predictor of all-cause mortality and cardiovascular death in the general population. Currently, there are three hs-cTn assays available. Roche hs-cTnT assay (Roche Diagnostics, Mannheim, Germany) and Abbott hs-cTnl assay (Abbott, Chicago, United States) have been used for several years. Numerous studies focused on diagnostic performance and clinical utility of hs-cTn determination in patients with ACS and in the general population [13-18]. Recently, a novel hs-cTnl assay by Siemens (Siemens Healthineers, Eschborn, Germany), following referred to as ADVIA Centaur hs-cTnl, has become available. In a study by Boeddinghaus and colleagues, this novel hscTnl assay showed high diagnostic accuracy and clinical performance in diagnosing ACS and a comparable performance to the aforementioned hs-cTn assays [8].

Patients with diabetes mellitus show elevated plasma cTn concentrations and various diabetes associated factors contributing to the increased CVD risk including hyperglycemia, dyslipidemia and alterations in the hemostasis system [19-22]. A useful tool to assess early forms of atherosclerosis in clinical practice is the determination of carotid intima-media thickness (cIMT) by high-resolution ultrasound. cIMT increases with age and is known to be larger in patients with T2DM compared to healthy controls [23, 24]. While CIMT is a risk marker for future CVD events in the general population [24], cIMT progression was not associated with future cardiovascular risk in patients with diabetes [25].

We now addressed the contribution of hs-cTnl, measured by the novel ADVIA Centaur hs-cTnl assay, to cardiovascular risk which was assessed by cIMT in T2DM. We furthermore compared this to classical clinical CVD risk factors.

\section{Methods}

\section{Cohort}

234 patients with T2DM were included in the analysis. 100 of the patients were women (43\%) and 134 men (58\%) with a median age of 65 years (57-71) and the median duration of diabetes mellitus was 10 years (6-17). Anthropometric data, clinical characteristics and laboratory parameters are shown in $>$ Table 1 . The study was conducted at the Department of Internal Medicine of the University Hospital Tübingen. Data were obtained from inpatients taking part at a diabetes mellitus education program according to recent guidelines [26]. Patients with type 1 diabetes mellitus and those who had a record of diabetes secondary to other causes (e. g. genetic defects of beta cell function, monogenic forms of diabetes, diabetes mellitus due to other pancreatic diseases) were excluded from the study. A detailed history including demographics and time since diagnosis of T2DM was taken from all subjects, followed by routine laboratory testing and physical examination including anthropometric data acquisition such as body mass index. In addition, patients were asked for history or presence of macrovascular disease (coronary artery disease, peripheral vascular disease, cerebrovascular disease). Blood pressure was measured according to Riva-Rocci by auscultation after resting for $10 \mathrm{~min}$ in sitting position. The study was conducted in accordance with the ethical standards of the Declaration of Helsinki from 1964 and its later amendments. Written informed consent was obtained from all study participants. The local ethics committee approved the protocol.

\section{Laboratory assessment and clinical parameters}

Determination of hs-cTnl was performed on an ADVIA Centaur XPT Immunoassay System (Siemens Healthineers, Eschborn, Germany) using re-thawed lithium-heparinized plasma samples. The limit of detection is indicated according to the manufacturer as $2.21 \mathrm{ng} / \mathrm{l}$, the limit of blank as $0.90 \mathrm{ng} / \mathrm{l}$ and the $99^{\text {th }}$ percentile concentrations are reported as 57 and $37 \mathrm{ng} / \mathrm{l}$ for men and women, respectively. All other clinical chemistry analyses (i. e. creatinine [enzymatic method], glucose [hexokinase method], total cholesterol, highdensity lipoprotein-cholesterol [HDL-cholesterol], low-density lipoprotein-cholesterol [LDL-cholesterol], triglycerides and C-reactive protein) were measured on an ADVIA Chemistry XPT analyzer following manufacturer's instructions, except for apolipoprotein $\mathrm{Al}$ and apolipoprotein B, which were measured on a BN ProSpec System (Siemens Healthineers). All laboratory parameters were measured after an overnight fasting period. Glycated hemoglobin (HbA1c) was measured using EDTA whole blood on a Tosoh G8 HPLC Analyzer (Tosoh Bioscience, Griesheim, Germany). Estimated glomerular filtration rate (eGFR) was calculated using a modified MDRD (Modification of Diet in Renal Disease)-formula based on plasma creatinine concentration, gender and age [27].

\section{Measurement of carotid intima-media thickness}

The cIMT of the common carotid artery was measured using highresolution ultrasound with a $10-13 \mathrm{MHz}$-linear array transducer (AU5 Harmonic, Esaote Biomedica, Munich, Germany). Ultrasound of both left and right common carotid artery was performed according to the European Mannheim carotid intima-media thickness consensus [28]. In detail, B-mode cIMT was assessed on the far wall of the artery approximately $10 \mathrm{~mm}$ proximal to the carotid bulb as described before [29].

\section{Statistical analysis}

Continuous data were presented as mean \pm standard deviation for normally distributed variables and as median (lower quartile - upper quartile) for non-normally distributed variables. Normal distribution was tested for all continuous variables using the Shapiro-Wilk- 
- Table 1 Patients' clinical and laboratory characteristics $(n=234)$.

\begin{tabular}{|c|c|c|c|c|}
\hline Variable & Total cohort $(n=234)$ & Men $(n=134)$ & Women $(n=100)$ & $\begin{array}{l}\text { p-value men vs. } \\
\text { women }\end{array}$ \\
\hline Age, years & $65(57-71)$ & $65(57-71)$ & $64(56-71)$ & 0.9566 \\
\hline Body-Mass-Index, $\mathrm{kg} / \mathrm{m}^{2}$ & $32.8(28.4-39.0)$ & $33.3(29.0-38.8)$ & $31.4(27.0-39.3)$ & 0.1070 \\
\hline Time since diabetes diagnosis, years & $10(6-17)$ & $11(6-16)$ & $9(5-18)$ & 0.7619 \\
\hline Systolic blood pressure, $\mathrm{mmHg}$ & $130(122-140)$ & $130(120-140)$ & $130(125-140)$ & 0.1546 \\
\hline Diastolic blood pressure, $\mathrm{mmHg}$ & $80(75-80)$ & $80(75-80)$ & $80(75-82)$ & 0.3778 \\
\hline Glucose (fasting), mmol/l & $8.6(7.2-10.6)$ & $8.6(7.2-11.0)$ & $8.4(6.9-10.0)$ & 0.1626 \\
\hline Glycated haemoglobin (HbA1c), \% & $7.6(6.9-9.1)$ & $7.7(6.9-9.2)$ & $7.6(6.8-8.9)$ & 0.3062 \\
\hline Glycated haemoglobin (HbA1c), mmol/mol & $59.6(51.9-76.0)$ & $60.7(51.9-76.0)$ & $59.6(50.8-73.8)$ & \\
\hline Creatinine, mg/dl & $0.8(0.7-1.0)$ & $0.9(0.8-1.2)$ & $0.7(0.6-0.9)$ & $<0.0001$ \\
\hline eGFR (MDRD), $\mathrm{ml} / \mathrm{min} / 1,73 \mathrm{~m}^{2}$ & $84(61-100)$ & 84 (60-99) & $83(62-102)$ & 0.8781 \\
\hline Triglycerides, mmol/l & $1.9(1.4-2.4)$ & $1.9(1.3-2.2)$ & $1.9(1.4-2.7)$ & 0.5163 \\
\hline Total cholesterol, mmol/l & $4.6(4.0-5.3)$ & $4.5(3.8-5.2)$ & $4.8(4.1-5.5)$ & 0.0048 \\
\hline HDL-Cholesterol, mmol/l & $1.1(0.9-1.3)$ & $1.1(0.9-1.3)$ & $1.2(1.0-1.4)$ & 0.0029 \\
\hline LDL-Cholesterol, mmol/l & $3.0(2.4-3.9)$ & $3.0(2.3-3.8)$ & $3.0(2.6-4.1)$ & 0.0523 \\
\hline Apolipoprotein Al, mg/dl & $149 \pm 22$ & $144 \pm 20$ & $154 \pm 22$ & 0.0002 \\
\hline Apolipoprotein B, mg/dl & $95(80-112)$ & $93(77-110)$ & $99(82-118)$ & 0.0379 \\
\hline Apolipoprotein B/Apolipoprotein AI & $0.6(0.5-0.8)$ & $0.6(0.5-0.8)$ & $0.6(0.5-0.8)$ & 0.9065 \\
\hline C-reactive Protein, mg/dl & $0.33(0.09-0.67)$ & $0.23(0.07-0.62)$ & $0.45(0.16-0.91)$ & 0.0030 \\
\hline Macroangiopathy", \% (n) & $31(74)$ & $41(56)$ & $18(18)$ & $<0.0001$ \\
\hline Smoking, \% (n) & $15(35)$ & $16(21)$ & $14(14)$ & 0.7405 \\
\hline Carotid intima-media thickness, mm & $0.75(0.70-0.95)$ & $0.80(0.70-0.95)$ & $0.73(0.65-0.85)$ & 0.0021 \\
\hline High-sensitivity cardiac troponin I ${ }^{*}, \mathrm{ng} / \mathrm{l}$ & $4.0(2.0-10.0)$ & $5.0(3.0-12.0)$ & $3.0(2.0-6.8)$ & 0.0005 \\
\hline
\end{tabular}
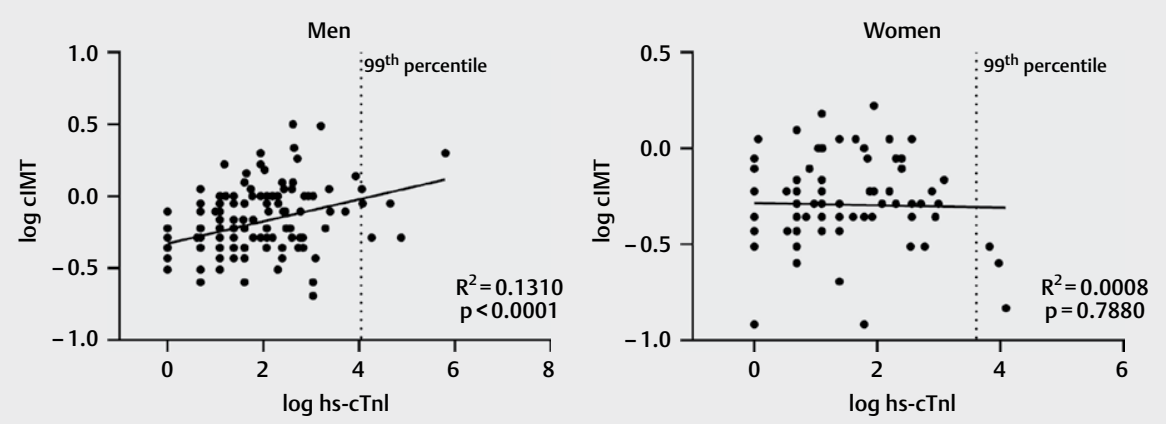

Fig. 1 Association of high-sensitivity cardiac troponin I (hs-cTnl) with carotid intima media thickness in type 2 diabetes. Shown are results of linear regression and correlation analyses separated by gender. A vertical line indicates the gender-specific $9^{9^{\text {th }}}$ percentile cut-off concentration for hs-cTnl.

W test. Non-normal distributed data were transformed with a logarithmic function prior to statistical analyses. The non-parametric rank sum test was used to compare two groups. The association of hs-cTnl results with clinical and laboratory variables were analysed using simple and multiple linear regression analysis. A p-value $<0.05$ was considered to indicate significant difference. For the selection of variables in the multiple regression analysis, stepwise linear regression (forward selection) was performed. Statistical analyses were conducted with JMP 14 (SAS Institute, Cary, United
States). - Figure 1 was created using GraphPad Software (version 8; GraphPad Software Inc., San Diego, United States).

\section{Results}

\section{High-sensitivity cardiac troponin I in patients with type 2 diabetes mellitus}

Plasma hs-cTnl concentrations were measurable in 226 patients (97\%) with a median hs-cTnl concentration of $4.0 \mathrm{ng} / \mathrm{l}(2.0-10.0)$ 
as presented in $>$ Table 1. Among all T2DM patients, 9 individuals (4\%) had hs-cTnl concentrations above the $99^{\text {th }}$ gender-specific percentile. 217 individuals (93\%) had concentrations below these thresholds and 72 individuals ( $31 \%$ ) showed concentrations below the limit of detection $(2.21 \mathrm{ng} / \mathrm{ml})$ of which 8 individuals $(3 \%)$ had concentrations below the limit of blank $(0.90 \mathrm{ng} / \mathrm{l})$. Median concentration of hs-cTnl was higher in men compared to women (5.0 vs. $3.0 \mathrm{ng} / \mathrm{l} ; \mathrm{p}=0.0005)$. Total cholesterol (4.5 vs. $4.8 \mathrm{mmol} / \mathrm{l}$; $\mathrm{p}=0.0048)$ and HDL-cholesterol ( $1.1 \mathrm{vs} .1 .2 \mathrm{mmol} / \mathrm{l} ; \mathrm{p}=0.0025$ ) concentrations were lower in men compared to women. In addition, mean concentration of apolipoprotein $\mathrm{Al}$ (154 vs. $144 \mathrm{mg} / \mathrm{dl}$; $p=0.0002$ ), median concentrations of apolipoprotein B (99 vs. $93 \mathrm{mg} / \mathrm{dl} ; \mathrm{p}=0.0379)$ and C-reactive protein $(0.45 \mathrm{vs} .0 .23 \mathrm{mg} / \mathrm{dl}$; $\mathrm{p}=0.0030$ ) were higher in women than in men. Although median creatinine concentration ( 0.9 vs. $0.7 \mathrm{mg} / \mathrm{dl}$; $p<0.0001)$ was significantly higher in men compared to women, the calculated eGFR (MDRD) did not differ between genders $(p=0.8781)$.
137 patients (59\%) used lipid-lowering drugs. Metformin was taken by 156 patients (67\%) and 137 patients (59\%) were on insulin therapy. 35 patients (15\%) reported to be smokers and 75 patients (35\%) had a history of smoking. Median cIMT was $0.75 \mathrm{~mm}$, ranging from $0.70-0.95 \mathrm{~mm}$ with higher cIMT in men compared to women ( 0.80 vs. $0.73 \mathrm{~mm} ; \mathrm{p}=0.0021$ ). Macrovascular disease was present in 56 of the men (41\%) and in 18 of the women (18\%) with T2DM, with a significant difference comparing the presence of macrovascular disease by gender $(p<0.0001)$.

\section{Associations between hs-cTnl concentrations and markers of cardiovascular risk and vascular damage}

For the understanding of the association of hs-cTnl concentrations with cIMT and traditional cardiovascular risk factors, we performed simple linear regression analyses ( $\triangleright$ Table 2 ). In the entire cohort, hs-cTnl was significantly associated with age (effect size $\beta_{\text {st }}=0.25$; $p=0.0001)$, gender $\left(\beta_{s t}=-0.24 ; p=0.0003\right)$, macrovascular disease $\left(\beta_{s t}=-0.23 ; p=0.0005\right)$, eGFR $\left(B_{s t}=-0.29 ; p<0.0001\right)$ and

- Table 2 Predictors of high-sensitivity cardiac troponin I in patients with type 2 diabetes. Shown are effect sizes $\left(B_{\mathrm{st}}\right)$ and p-values of univariate relationships between high-sensitivity cardiac troponin I and anthropometric or laboratory parameters in the entire cohort and separated by gender.

\begin{tabular}{|c|c|c|c|}
\hline \multirow[t]{2}{*}{ Variable } & \multicolumn{3}{|c|}{ High-sensitivity cardiac troponin I } \\
\hline & Total cohort $(n=226)$ & Men $(n=130)$ & Women $(n=96)$ \\
\hline Gender (female $=0$, male $=1$ ) & $\begin{array}{l}-0.24 \\
\mathbf{p}=\mathbf{0 . 0 0 0 3}\end{array}$ & - & - \\
\hline Age, years & $\begin{array}{l}0.25 \\
\mathbf{p}=\mathbf{0 . 0 0 0 1}\end{array}$ & $\begin{array}{l}0.29 \\
p=0.0009\end{array}$ & $\begin{array}{l}0.24 \\
p=0.0178\end{array}$ \\
\hline Body-Mass-Index, kg/m² & $\begin{array}{l}-0.01 \\
p=0.8925\end{array}$ & $\begin{array}{l}-0.12 \\
p=0.1831\end{array}$ & $\begin{array}{l}0.09 \\
p=0.4050\end{array}$ \\
\hline Time since diabetes diagnosis, years & $\begin{array}{l}0.06 \\
p=0.3872\end{array}$ & $\begin{array}{l}0.05 \\
p=0.5381\end{array}$ & $\begin{array}{l}0.07 \\
p=0.4736\end{array}$ \\
\hline Systolic blood pressure, $\mathrm{mmHg}$ & $\begin{array}{l}0.03 \\
p=0.6275\end{array}$ & $\begin{array}{l}0.02 \\
p=0.7816\end{array}$ & $\begin{array}{l}0.10 \\
p=0.3372\end{array}$ \\
\hline Diastolic blood pressure, $\mathrm{mmHg}$ & $\begin{array}{l}-0.07 \\
p=0.3192\end{array}$ & $\begin{array}{l}-0.07 \\
p=0.4054\end{array}$ & $\begin{array}{l}-0.01 \\
p=0.9066\end{array}$ \\
\hline Glucose (fasting), mmol/l & $\begin{array}{l}-0.05 \\
p=0.4953\end{array}$ & $\begin{array}{l}-0.05 \\
p=0.5508\end{array}$ & $\begin{array}{l}-0.08 \\
p=0.4375\end{array}$ \\
\hline Glycated haemoglobin, \% & $\begin{array}{l}-0.09 \\
p=0.1980\end{array}$ & $\begin{array}{l}-0.09 \\
p=0.2991\end{array}$ & $\begin{array}{l}-0.12 \\
p=0.2399\end{array}$ \\
\hline eGFR (MDRD), $\mathrm{ml} / \mathrm{min} / 1,73 \mathrm{~m}^{2}$ & $\begin{array}{l}-0.29 \\
\mathbf{p}<0.0001\end{array}$ & $\begin{array}{l}-0.39 \\
\mathbf{p}<0.0001\end{array}$ & $\begin{array}{l}-0.18 \\
p=0.0735\end{array}$ \\
\hline Triglycerides, mmol/l & $\begin{array}{l}0.01 \\
p=0.9988\end{array}$ & $\begin{array}{l}-0.02 \\
p=0.8433\end{array}$ & $\begin{array}{l}0.04 \\
p=0.6832\end{array}$ \\
\hline Total cholesterol, mmol/l & $\begin{array}{l}-0.03 \\
p=0.6199\end{array}$ & $\begin{array}{l}0.02 \\
p=0.8621\end{array}$ & $\begin{array}{l}-0.01 \\
p=0.8952\end{array}$ \\
\hline HDL-Cholesterol, mmol/l & $\begin{array}{l}0.03 \\
p=0.6137\end{array}$ & $\begin{array}{l}0.18 \\
\mathbf{p}=\mathbf{0 . 0 4 5 2}\end{array}$ & $\begin{array}{l}-0.03 \\
p=0.7792\end{array}$ \\
\hline LDL-Cholesterol, mmol/I & $\begin{array}{l}-0.02 \\
p=0.7448\end{array}$ & $\begin{array}{l}0.02 \\
p=0.8377\end{array}$ & $\begin{array}{l}-0.02 \\
p=0.8672\end{array}$ \\
\hline Macrovascular disease (yes $=1$ ) & $\begin{array}{l}-0.23 \\
\mathbf{p}=\mathbf{0 . 0 0 0 5}\end{array}$ & $\begin{array}{l}-0.24 \\
p=0.0050\end{array}$ & $\begin{array}{l}-0.07 \\
p=0.4993\end{array}$ \\
\hline Smoking (yes = 1 ) & $\begin{array}{l}0.09 \\
p=0.6156\end{array}$ & $\begin{array}{l}-0.07 \\
p=0.6959\end{array}$ & $\begin{array}{l}0.17 \\
p=0.7896\end{array}$ \\
\hline C-reactive Protein, mg/dl & $\begin{array}{l}0.09 \\
p=0.1561\end{array}$ & $\begin{array}{l}0.17 \\
p=0.0531\end{array}$ & $\begin{array}{l}0.08 \\
p=0.4329\end{array}$ \\
\hline Carotid intima-media thickness, mm & $\begin{array}{l}0.27 \\
\mathbf{p}<0.0001\end{array}$ & $\begin{array}{l}0.34 \\
\mathbf{p}<0.0001\end{array}$ & $\begin{array}{l}0.05 \\
p=0.6142\end{array}$ \\
\hline
\end{tabular}


- Table 3 Multiple linear regression analysis of associations with highsensitivity cardiac troponin I in patients with type 2 diabetes. Shown are effect sizes $\left(B_{s t}\right)$ and $p$-values of multivariable regression analyses with all indicated variables.

\begin{tabular}{|l|c|c|c|}
\hline \multirow{2}{*}{ Variable } & \multicolumn{3}{|c|}{ High-sensitivity cardiac troponin I } \\
\cline { 2 - 4 } & $\begin{array}{l}\text { Total cohort } \\
(\mathbf{n = 2 2 6})\end{array}$ & $\begin{array}{l}\text { Men } \\
(\mathbf{n = 1 3 0 )}\end{array}$ & $\begin{array}{l}\text { Women } \\
(\mathbf{n = 9 6 )}\end{array}$ \\
\hline Gender (female =0, male =1) & -0.22 & - & - \\
& $\mathbf{0 . 0 0 0 7}$ & & \\
\hline Age, years & 0.11 & 0.04 & 0.24 \\
& 0.1064 & 0.7094 & $\mathbf{0 . 0 2 8 0}$ \\
\hline Glycated haemoglobin, mmol/ & -0.12 & -0.10 & -0.18 \\
mol & 0.0619 & 0.2384 & 0.0927 \\
\hline eGFR, ml/min/1.73 m & -0.20 & -0.24 & -0.14 \\
& $\mathbf{0 . 0 0 2 8}$ & $\mathbf{0 . 0 1 2 6}$ & 0.1764 \\
\hline Macrovascular disease (yes=1) & -0.09 & -0.07 & -0.06 \\
& 0.1957 & 0.4142 & 0.5490 \\
\hline C-reactive Protein & 0.14 & 0.12 & 0.17 \\
& $\mathbf{0 . 0 2 3 4}$ & 0.1317 & 0.1176 \\
\hline Carotid intima-media & 0.12 & 0.24 & -0.09 \\
thickness, mm & 0.0837 & $\mathbf{0 . 0 0 8 1}$ & 0.3850 \\
\hline
\end{tabular}

cIMT $\left(B_{\mathrm{st}}=0.27 ; \mathrm{p}<0.0001\right)$. As clMT interacted with gender on hs$c \operatorname{Tnl}(p=0.0256)$, subsequent analyses were performed separately for both genders. In men, significant associations of hs-cTnl with age $\left(\beta_{\text {st }}=0.29 ; p=0.0009\right)$, HDL-cholesterol $\left(\beta_{s t}=0.18 ; p=0.0452\right)$, $\operatorname{eGFR}\left(\beta_{\mathrm{st}}=-0.39 ; \mathrm{p}<0.0001\right)$ and $\mathrm{cIMT}\left(\aleph_{\mathrm{st}}=0.34 ; \mathrm{p}<0.0001\right)$ were detected in univariate analysis. In women, only age was significantly associated with hs-cTnl ( $\left.ß_{\mathrm{st}}=0.24 ; \mathrm{p}=0.0178\right)$. CIMT and eGFR showed no significant association with hs-cTnl in women ( $\triangleright$ Table $\mathbf{2}$ and $>$ Figure 1). To identify independent predictors of hs-cTnl multiple linear regression analyses were performed. Therefore, stepwise regression analysis was utilized to identify variables for the multivariable regression model ( $\triangleright$ Table 3$)$. In the final analyses only gender $\left(\beta_{\mathrm{st}}=-0.22 ; \mathrm{p}=0.0007\right)$, eGFR $\left(\beta_{\mathrm{st}}=-0.20\right.$; $p=0.0028)$ and $C$-reactive protein $\left(\beta_{s t}=0.14 ; p=0.0234\right)$ were significantly associated with hs-cTnl in the entire cohort. Analysis separated by gender revealed eGFR ( $\left.\beta_{\mathrm{st}}=-0.24 ; \mathrm{p}=0.0126\right)$ and $\mathrm{cIMT}$ $\left(B_{\mathrm{st}}=0.24 ; \mathrm{p}=0.0081\right)$ to be independently associated with hs-cT$\mathrm{nl}$ in men ( $\triangleright$ Table 3). Adjusting for the same covariates in women, age $\left(B_{\mathrm{st}}=0.24 ; \mathrm{p}=0.0280\right.$ ) remained as a single factor significantly associated with hs-cTnl. Finally, the influence of traditional cardiovascular risk factors, such as body mass index, systolic blood pressure, LDL-cholesterol and smoking on the final model was tested. CIMT ( $\left.\beta_{s t}=0.25 ; p=0.0067\right)$ and eGFR $\left(B_{s t}=-0.23 ; p=0.0169\right)$ still remained significantly associated with hs-cTnl in men and, again, only the association between hs-cTnl and age in women $\left(B_{\mathrm{st}}=0.27 ; \mathrm{p}=0.0234\right)$.

\section{Discussion}

We here investigated links between high-sensitivity cardiac troponin I (hs-cTnl) and cardiovascular risk factors and early signs of arteriosclerotic disease in patients with type 2 diabetes. Our results revealed important gender-specific differences: Men showed higher cIMT compared to women as shown before [29, 30]. In men, cIMT was significantly associated with elevated hs-cTnl concentrations, a marker of subclinical myocardial damage, independently of traditional CVD risk factors, such as age, body mass index, smoking and LDL-cholesterol. The association between subclinical myocardial damage and cIMT was independent of the presence of macrovascular disease. Interestingly, women did not show the association between cIMT and hs-cTnl, whereby cIMT gender differences, to the detriment of men, has shown early before [31]. Age was the single independently associated risk factor of hs-cTnl in women with T2DM. This is in line with several other studies reporting age as the main risk factor of cardiovascular death in women [32-34]. A previous study investigated the association of Abbott hs-cTnl and an additional marker of vascular damage by using brachial-arterial pulse wave velocity [35]. A significant association between hs-cTnl and brachial-arterial pulse wave velocity (ba-PWV), a marker of arterial stiffness, was demonstrated, but not with cIMT. Unfortunately, the study did not report gender-specific associations. Nevertheless, their results are in line with our findings in the entire cohort.

T2DM itself is associated with an increased cIMT and gender differences of cIMT are also well documented [23, 36-38]. Consequently, gender-specific risk stratification in T2DM should be performed, which may have important implications in prognosis and potentially the treatment of these patients.

Further interesting findings were observed in our study: the majority of T2DM patients in our study showed measurable hs-cTnl concentrations below the gender-specific $99^{\text {th }}$ percentile. Hs-cTn assays are frequently used today and provide results with strongly increased diagnostic accuracy even at very low troponin concentrations within the reference interval [8]. Several other studies consistently showed that elevated troponin plasma concentrations are present in patients with T2DM patients compared to non-diabetics either using older troponin assays or hs-cTn assays $[39,40]$. It was shown that even slightly increased hs-cTn concentrations, still within the reference interval, are associated with subclinical myocardial damage and are therefore predictive for future cardiovascular events in the general population and in patients attending an emergency room without obvious cardiac diseases $[13,14,16]$. This link emphasizes the need to include hs-cTn assays in the risk stratification of patients with T2DM to identify individuals at increased risk for cardiovascular events.

We found increased hs-cTnl concentrations in men compared to women as it was previously demonstrated with the introduction of the first hs-cTn assays gender-specific differences of hs-cTn concentrations $[41,42]$. In a recent study, Boeddinghaus and colleagues confirmed gender-specific differences determined by the same hs-cTnl assay we used in our study in patients presenting with suspicion of acute coronary syndrome to the emergency unit [8]. We observed nearly 2 -fold higher hs-cTnl in men as it was consistently reported by the aforementioned studies. It is now widely accepted that troponin concentrations, in line with other biomarkers, are influenced by gender and that gender-specific $99^{\text {th }}$ percentile must be taken into account $[5,11]$.

Renal function, determined by eGFR (MDRD), was found to be another parameter, which is associated with hs-cTnl in our study. And again, this association was only true in men. Similar findings were reported in previous publications showing significant association between renal function and hs-cTn concentrations in patients 
admitting the emergency room $[43,44]$. Studies using hs-cTnT and hs-cTnl assays in parallel showed stronger correlation of renal function to hs-cTnT compared to hs-cTnl $[43,44]$. Unfortunately, we did not measure hs-cTnT in our study to focus on this specific issue. In our study, the majority of T2DM patients had an eGFR within the reference interval. It is therefore not possible to make detailed statements to the association of hs-cTnl and chronic kidney disease, especially, since we do not have an additional marker for early nephropathy such as albumin in urine measurement. However, there is evidence that hs-cTnl is not increased in patients with dialysis-dependent renal failure. In a recent study it was demonstrated that hs-cTnl concentrations were below the $99^{\text {th }}$ percentile in the majority of these patients [45].

Strength of our study is the observation in a real-world clinical setting in a well characterized cohort of patients with T2DM. We assessed subclinical myocardial damage using a novel hs-cTnl assay and investigated the association to cIMT, an established marker of atherosclerosis. Limitations of the study include the cross-sectional design and the lack of detailed data about cardiac parameters (i. e. cardiac function). Regarding cIMT as an early marker of subclinical atherosclerosis, a point of limitation is that we used this tool in a cohort including patients with known macrovascular disease. Nevertheless, we are looking at cIMT as a continuous variable and an increment of CIMT is associated with an elevated CVD risk [24]. We did not examine more distal segments of the carotid artery (i.e. the internal carotid artery) or plaque burden. Latter is known to be an important marker of advanced atherosclerosis [46]. Regarding laboratory parameters, the MDRD formula for estimating GFR used in this study is not accurate for values within the normal range and results should therefore be carefully interpreted. Finally, results of the study need to be validated in larger cohorts, ideally combined with follow-up measurements to investigate the prognostic utility of the association between hs-cTnl and cIMT.

In conclusion, the present study in a real-world clinical setting demonstrated that the majority of patients with T2DM showed measurable hs-cTnl concentrations using the novel ADIVA centaur hs-cTnl assay. CIMT was significantly associated with hs-cTnl in men and is therefore suitable as predictor of subclinical myocardial damage in men, but not in women.

\section{Acknowledgements}

We gratefully thank all participants of the study and Marjo Graf, Susanne Faix, Isolde Riedlinger and Ann Katrin Horlacher for their excellent technical assistance.

\section{Conflict of Interest}

The authors declare that they have no conflict of interest.

\section{References}

[1] Everett BM, Brooks MM, Vlachos HE et al. Troponin and cardiac events in stable ischemic heart disease and diabetes. N Engl J Med 2015; 373 : 610-620. DOI: 10.1056/NEJMoa1415921

[2] Diabetes mellitus: a major risk factor for cardiovascular disease. A joint editorial statement by the American Diabetes Association; The National Heart, Lung, and Blood Institute; The Juvenile Diabetes Foundation International; The National Institute of Diabetes and Digestive and Kidney Diseases; and The American Heart Association. Circulation 1999; 100: 1132-1133

[3] Lloyd-Jones DM, Leip EP, Larson MG et al. Prediction of lifetime risk for cardiovascular disease by risk factor burden at 50 years of age. Circulation 2006; 113: 791-798. DOI: 10.1161/circulationaha.105.548206

[4] Emerging Risk Factors C, Sarwar N, Gao P et al. Diabetes mellitus, fasting blood glucose concentration, and risk of vascular disease: $\mathrm{A}$ collaborative meta-analysis of 102 prospective studies. Lancet 2010; 375: 2215-2222. DOI: 10.1016/S0140-6736(10)60484-9

[5] Thygesen K, Alpert JS, Jaffe AS et al. Fourth universal definition of myocardial infarction (2018). Eur Heart J 2019; 40: 237-269. DOI: 10.1093/eurheartj/ehy462

[6] Thygesen K, Mair ], Katus $\mathrm{H}$ et al. Recommendations for the use of cardiac troponin measurement in acute cardiac care. Eur Heart J 2010; 31: 2197-2204. DOI: 10.1093/eurheartj/ehq251

[7] Damman P, van 't Hof AW, Ten Berg JM et al. 2015 ESC guidelines for the management of acute coronary syndromes in patients presenting without persistent ST-segment elevation: Comments from the Dutch ACS working group. Neth Heart J 2017; 25: 181-185. DOI: 10.1007/ s12471-016-0939-y

[8] Boeddinghaus ], Twerenbold R, Nestelberger T et al. Clinical validation of a novel high-sensitivity cardiac troponin I assay for early diagnosis of acute myocardial infarction. Clin Chem 2018; 64: 1347-1360. DOI: 10.1373/clinchem.2018.286906

[9] McCarthy CP, Raber I, Chapman AR et al. Myocardial injury in the era of high-sensitivity cardiac troponin assays: A practical approach for clinicians. JAMA Cardiol. 2019; DOI: 10.1001/jamacardio.2019.2724

[10] Goodman SG, Steg PG, Eagle KA et al. The diagnostic and prognostic impact of the redefinition of acute myocardial infarction: Lessons from the Global Registry of Acute Coronary Events (GRACE). Am Heart J 2006; 151: 654-660. DOI: 10.1016/j.ahj.2005.05.014

[11] Apple FS, Sandoval Y, Jaffe AS et al. Cardiac troponin assays: Guide to understanding analytical characteristics and their impact on clinical care. Clin Chem 2017; 63: 73-81. DOI: 10.1373/clinchem.2016.255109

[12] Frankenstein L, Wu AH, Hallermayer $\mathrm{K}$ et al. Biological variation and reference change value of high-sensitivity troponin $\mathrm{T}$ in healthy individuals during short and intermediate follow-up periods. Clin Chem 2011; 57: 1068-1071. DOI: 10.1373/clinchem.2010.158964

[13] Willeit P, Welsh P, Evans JDW et al. High-sensitivity cardiac troponin concentration and risk of first-ever cardiovascular outcomes in 154052 participants. J Am Coll Cardiol 2017; 70: 558-568. DOI: 10.1016/j. jacc.2017.05.062

[14] Blankenberg S, Salomaa V, Makarova N et al. Troponin I and cardiovascular risk prediction in the general population: The BiomarCaRE consortium. Eur Heart J 2016; 37: 2428-2437. DOI: 10.1093/ eurheartj/ehw172

[15] Seliger SL, Hong SN, Christenson RH et al. High-sensitive cardiac troponin $\mathrm{T}$ as an early biochemical signature for clinical and subclinical heart failure: MESA (Multi-Ethnic Study of Atherosclerosis). Circulation 2017; 135: 1494-1505. DOI: 10.1161/circulationaha.116.025505

[16] Zhu K, Knuiman M, Divitini M et al. High-sensitivity cardiac troponin I and risk of cardiovascular disease in an Australianpopulation-based cohort. Heart 2018; 104: 895-903. DOI: 10.1136/ heartjnl-2017-312093 
[17] Welsh P, Preiss D, Shah ASV et al. Comparison between high-sensitivity cardiac troponin T and cardiac troponin I in a large general population cohort. Clin Chem 2018; 64: 1607-1616. DOI: 10.1373/ clinchem.2018.292086

[18] Rubini Gimenez M, Twerenbold R, Reichlin T et al. Direct comparison of high-sensitivity-cardiac troponin I vs. T for the early diagnosis of acute myocardial infarction. Eur Heart J 2014; 35: 2303-2311. DOI: 10.1093/eurheartj/ehu188

[19] Hallen J, Johansen OE, Birkeland KI et al. Determinants and prognostic implications of cardiac troponin T measured by a sensitive assay in type 2 diabetes mellitus. Cardiovasc Diabetol 2010; 9: 52. DOI: 10.1186/1475-2840-9-52

[20] Festa A, D'Agostino R Jr., Tracy RP et al. Elevated levels of acute-phase proteins and plasminogen activator inhibitor- 1 predict the development of type 2 diabetes: the insulin resistance atherosclerosis study. Diabetes 2002; 51: 1131-1137

[21] Kannel WB. Lipids, diabetes, and coronary heart disease: Insights from the Framingham Study. Am Heart J 1985; 110: 1100-1107

[22] Balletshofer BM, Rittig K, Enderle MD et al. Endothelial dysfunction is detectable in young normotensive first-degree relatives of subjects with type 2 diabetes in association with insulin resistance. Circulation 2000; 101: 1780-1784

[23] Brohall G, Oden A, Fagerberg B. Carotid artery intima-media thickness in patients with Type 2 diabetes mellitus and impaired glucose tolerance: A systematic review. Diabet Med 2006; 23: 609-616. DOI: 10.1111/j.1464-5491.2005.01725.x

[24] Lorenz MW, Markus HS, Bots ML et al. Prediction of clinical cardiovascular events with carotid intima-media thickness: A systematic review and meta-analysis. Circulation 2007; 115: 459-467. DOI: 10.1161/ circulationaha.106.628875

[25] Lorenz MW, Price JF, Robertson C et al. Carotid intima-media thickness progression and risk of vascular events in people with diabetes: Results from the PROG-IMT collaboration. Diabetes Care 2015; 38: 19211929. DOI: $10.2337 / \mathrm{dc} 14-2732$

[26] Dallosso H, Yates T, Mani H et al. Movement through Active Personalised engagement (MAP) - a self-management programme designed to promote physical activity in people with multimorbidity: study protocol for a randomised controlled trial. Trials 2018; 19: 576. DOI: 10.1186/s13063-018-2939-2

[27] Levey AS, Coresh J, Greene T et al. Expressing the Modification of Diet in Renal Disease Study equation for estimating glomerular filtration rate with standardized serum creatinine values. Clin Chem 2007; 53 : 766-772. DOI: 10.1373/clinchem.2006.077180

[28] Touboul PJ, Hennerici MG, Meairs S et al. Mannheim carotid intimamedia thickness and plaque consensus (2004-2006-2011). An update on behalf of the advisory board of the 3rd, 4th and 5th watching the risk symposia, at the 13th, 15th and 20th European Stroke Conferences, Mannheim, Germany, 2004, Brussels, Belgium, 2006, and Hamburg, Germany, 2011. Cerebrovasc Dis 2012; 34: 290-296. DOI: $10.1159 / 000343145$

[29] Randrianarisoa E, Rietig R, Jacob S et al. Normal values for intimamedia thickness of the common carotid artery - an update following a novel risk factor profiling. Vasa 2015; 44: 444-450. DOI: 10.1024/0301-1526/a000467

[30] Chambless LE, Folsom AR, Davis $V$ et al. Risk factors for progression of common carotid atherosclerosis: The Atherosclerosis Risk in Communities Study, 1987-1998. Am J Epidemiol 2002; 155: 38-47

[31] O'Leary DH, Polak JF, Kronmal RA et al. Distribution and correlates of sonographically detected carotid artery disease in the Cardiovascular Health Study. The CHS Collaborative Research Group. Stroke 1992; 23: $1752-1760$
[32] Mosca L, Benjamin E], Berra K et al. Effectiveness-based guidelines for the prevention of cardiovascular disease in women--2011 update: A guideline from the american heart association. Circulation 2011; 123 : 1243-1262. DOI: 10.1161/CIR.0b013e31820faaf8

[33] GBD 2013 Mortality and Causes of Death Collaborators Global, regional, and national age-sex specific all-cause and cause-specific mortality for 240 causes of death, 1990-2013: a systematic analysis for the Global Burden of Disease Study 2013. Lancet 2015; 385: 117-171. DOI: 10.1016/S0140-6736(14)61682-2

[34] Maffei S, Guiducci L, Cugusi L et al. Women-specific predictors of cardiovascular disease risk - new paradigms. Int J Cardiol 2019; DOI: 10.1016/j.ijcard.2019.02.005

[35] Yiu KH, Zhao CT, Chen Y et al. Association of subclinical myocardial injury with arterial stiffness in patients with type 2 diabetes mellitus. Cardiovasc Diabetol 2013; 12: 94. DOI: 10.1186/1475-2840-12-94

[36] Wagenknecht LE, D'Agostino RB Jr., Haffner SM et al. Impaired glucose tolerance, type 2 diabetes, and carotid wall thickness: The Insulin Resistance Atherosclerosis Study. Diabetes Care 1998; 21: 1812-1818

[37] Temelkova-Kurktschiev TS, Koehler C, Leonhardt W et al. Increased intimal-medial thickness in newly detected type 2 diabetes: Risk factors. Diabetes Care 1999; 22: 333-338

[38] Kablak-Ziembicka A, Przewlocki T, Tracz W et al. Gender differences in carotid intima-media thickness in patients with suspected coronary artery disease. Am J Cardiol 2005; 96: 1217-1222. DOI: 10.1016/j. amjcard.2005.06.059

[39] Segre CA, Hueb W, Garcia RM et al. Troponin in diabetic patients with and without chronic coronary artery disease. BMC Cardiovasc Disord 2015; 15: 72. DOI: 10.1186/s12872-015-0051-z

[40] Cavender MA, White WB, Jarolim P et al. Serial Measurement of High-Sensitivity Troponin I and Cardiovascular Outcomes in Patients With Type 2 Diabetes Mellitus in the EXAMINE Trial (Examination of Cardiovascular Outcomes With Alogliptin Versus Standard of Care). Circulation 2017; 135: 1911-1921. DOI: 10.1161/circulationaha. 116.024632

[41] Collinson PO, Heung YM, Gaze D et al. Influence of population selection on the 99th percentile reference value for cardiac troponin assays. Clin Chem 2012; 58: 219-225. DOI: 10.1373/ clinchem.2011.171082

[42] Apple FS, Simpson PA, Murakami MM. Defining the serum 99th percentile in a normal reference population measured by a high-sensitivity cardiac troponin I assay. Clin Biochem 2010; 43: 1034-1036. DOI: 10.1016/j.clinbiochem.2010.05.014

[43] Guclu T, Bolat S, Senes M et al. Relationship between high sensitivity troponins and estimated glomerular filtration rate. Clin Biochem 2016; 49: 467-471. DOI: 10.1016/j.clinbiochem.2015.12.012

[44] Twerenbold R, Wildi K, Jaeger C et al. Optimal cutoff levels of more sensitive cardiac troponin assays for the early diagnosis of myocardial infarction in patients with renal dysfunction. Circulation 2015; 131: 2041-2050. DOI: 10.1161/circulationaha.114.014245

[45] Artunc F, Haag S, Friedrich B et al. Performance of a novel high sensitivity cardiac troponin I assay in asymptomatic hemodialysis patients - evidence for sex-specific differences. Clin Chem Lab Med 2019; 57: 1261-1270. DOI: 10.1515/cclm-2018-1176

[46] Simon A, Megnien JL, Chironi G. The value of carotid intima-media thickness for predicting cardiovascular risk. Arterioscler Thromb Vasc Biol 2010; 30: 182-185. DOI: 10.1161/atvbaha.109.196980 